\title{
NOX4/Src regulates ANP secretion through activating ERK1/2 and Akt/GATA4 signaling in beating rat hypoxic atria
}

\author{
Cheng-zhe Wu ${ }^{1,2, *}$, Xiang Li ${ }^{1, *}$, Lan Hong ${ }^{1}$, Zhuo-na Han', Ying Liu' ${ }^{1}$, Cheng-xi Wei ${ }^{3, *}$, and Xun Cui ${ }^{1,4, *}$ \\ ${ }^{1}$ Department of Physiology, School of Medicine, Yanbian University, ${ }^{2}$ Institute of Clinical Medicine, Yanbian University, Yanji 133-002, ${ }^{3}$ Inner Mongolia Univer- \\ sity for Nationalities, Tongliao 028000, ${ }^{4}$ Cellular Function Research Center, Yanbian University, Yanji 133-002, China
}

\author{
ARTICLE INFO \\ Received September 11, 2020 \\ Revised December 24, 2020 \\ Accepted December 29, 2020 \\ *Correspondence \\ Xun Cui \\ E-mail: cuixun@ybu.edu.cn \\ Cheng-xi Wei \\ E-mail: weichengxi1224@163.com
}

\section{Key Words}

Atrial natriuretic peptide

Endothelin-1

Hypoxia

NADPH oxidase

Src tyrosine kinase

"These authors contributed equally to this work.

\begin{abstract}
Nicotinamide adenine dinucleotide phosphate oxidases (NOXs) are the major enzymatic source of reactive oxygen species (ROS). NOX2 and NOX4 are expressed in the heart but its role in hypoxia-induced atrial natriuretic peptide (ANP) secretion is unclear. This study investigated the effect of NOX on ANP secretion induced by hypoxia in isolated beating rat atria. The results showed that hypoxia significantly upregulated NOX4 but not NOX2 expression, which was completely abolished by endothelin-1 (ET-1) type A and B receptor antagonists BQ123 (0.3 $\mu \mathrm{M})$ and BQ788 $(0.3 \mu \mathrm{M})$. ET-1-upregulated NOX4 expression was also blocked by antagonists of secreted phospholipase A2 (sPLA2; varespladib, $5.0 \mu \mathrm{M}$ ) and cytosolic PLA2 (cPLA2; CAY10650, 120.0 nM), and ET-1-induced CPLA2 expression was inhibited by varespladib under normoxia. Moreover, hypoxia-increased ANP secretion was evidently attenuated by the NOX4 antagonist GLX351322 (35.0 $\mu \mathrm{M})$ and inhibitor of ROS N-Acetyl-D-cysteine (NAC, $15.0 \mathrm{mM}$ ), and hypoxia-increased production of ROS was blocked by GLX351322. In addition, hypoxia markedly upregulated Src expression, which was blocked by ET receptors, NOX4, and ROS antagonists. ET-1-increased Src expression was also inhibited by NAC under normoxia. Furthermore, hypoxiaactivated extracellular signal-regulated kinase $1 / 2$ (ERK1/2) and protein kinase $B$ (Akt) were completely abolished by Src inhibitor $1(1.0 \mu \mathrm{M})$, and hypoxia-increased GATA4 was inhibited by the ERK1/2 and Akt antagonists PD98059 (10.0 $\mu \mathrm{M})$ and LY294002 $(10.0 \mu \mathrm{M})$, respectively. However, hypoxia-induced ANP secretion was substantially inhibited by Src inhibitor. These results indicate that NOX4/Src modulated by ET-1 regulates ANP secretion by activating ERK1/2 and Akt/GATA4 signaling in isolated beating rat hypoxic atria.
\end{abstract}

\section{INTRODUCTION}

Accumulating evidence implicates reactive oxygen species (ROS)-dependent production of ROS in the pathophysiology of cardiovascular diseases, such as hypertension, cardiac ischemia/ reperfusion injury, myocardial infarction, and heart failure [1]. Nicotinamide adenine dinucleotide phosphate oxidases (NOXs), a major enzymatic source of ROS in the cardiovascular system, generate ROS in a highly regulated manner [2]. NOXs are multi- subunit transmembrane enzymes; two isoforms, including NOX2 and NOX4, are expressed in the heart [2,3]. NOX2 was found to be essential for angiotensin II-induced cardiac hypertrophy, as indicated by increased mRNA levels of molecular biomarkers, such as atrial natriuretic peptide (ANP) and $\beta$-myosin heavy chain, in mice [4]. NOX4 overexpression also plays an important role in mediating cardiac hypertrophy, fibrosis, and apoptosis in response to pressure overload, although the mechanism of action remains to be defined [5].

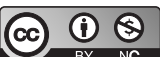

This is an Open Access article distributed under the terms of the Creative Commons Attribution Non-Commercial License, which permits unrestricted non-commercial use, distribution, and reproduction in any medium, provided the original work is properly cited. Copyright @ Korean J Physiol Pharmacol, pISSN 1226-4512, elSSN 2093-3827
Author contributions: C.Z.W. performed atrial perfused experiments. X.L. and L.H. performed WB analysis. Z.N.H. and Y.L. performed ANP measurement. X.C. and C.X.W. designed experiments and wrote the manuscript. 
The peptide hormone ANP is synthesized and secreted mainly from atrial myocytes in response to stretch and other stimuli [6]. In addition to cardiovascular roles, such as regulating natriuresis, diuresis, and vasodilation [7], ANP may also exhibit either antioxidant defense or pro-oxidant effects, depending on the experimental conditions and cell types [8]. Furthermore, it was demonstrated that ANP secretion is potently stimulated by hypoxia, resulting in cellular adaptation to hypoxia, protection of the heart against ischemia/reperfusion injury, reduced development of heart failure, and prevention of pathologic remodeling in dilated cardiomyopathy [9-11]. Recently, it was suggested that $G$ proteincoupled receptor (GPCR) agonists, such as endothelin-1 (ET-1), elicit ROS production by activating NOX to stimulate downstream signaling in cardiovascular disorders $[2,12]$. However, its role in atrial ANP secretion (especially under hypoxic conditions) is unclear. Therefore, the present study investigated the effect of endogenous ET-1 on the regulation of NOX expression and its role in ANP secretion in isolated beating rat hypoxic atria.

\section{METHODS}

\section{Reagents and antibodies}

Endothelin receptor type A (ETRA) antagonist BQ123 and endothelin receptor type $B$ (ETRB) antagonist BQ788 were purchased from Sigma (St. Louis, MO, USA). ET-1 (human, porcine) was purchased from Tocris Bioscience (Minneapolis, MN, USA). The NOX4 inhibitor GLX351322, secreted phospholipase (sPLA2) inhibitor varespladib, cytosolic PLA2 $\alpha$ (cPLA2 $\alpha$ ) inhibitor CAY10650, ROS inhibitor N-Acetyl-D-cysteine (NAC), Src antagonist Src inhibitor 1, extracellular signal-regulated kinase (ERK) antagonist PD98059, and protein kinase B (Akt) antagonist LY294002 were purchased from Med Chem Express (Monmouth Junction, NJ, USA). Anti-NOX4 and anti-GATA4 antibodies were obtained from Abcam (ab133303, ab85163, ab117529, and ab84593, respectively; Cambridge, UK). The anti-Src antibody was from Millipore (04-889; Burlington, MA, USA). Anti-NOX2 antibody was obtained from Bioss (bs-3889R; Beijing, China). Anti-Akt, phospho-Akt (S473), and $\beta$-actin antibodies were acquired from Bioworld Technology (BS1008, BS4006, and AP0060, respectively; Nan Jing, China). Anti-ERK1/2, phospho-ERK1/2 (Thr202/Tyr204), cPLA2, and phosho-cPLA2 (Ser505) antibodies were obtained from Affinity (AF0155, AF1015, AF6329, and AF3329, respectively; Chang Zhou, China).

\section{Preparation of perfused beating rat atria}

The experimental procedures in this study were approved by the Animal Care and Use Committee of Yanbian University and comply with animal welfare guidelines of the US National Institutes of Health. Sprague-Dawley rats (weighing 250-300 g, aged 18 weeks, female to male ratio of 3:7) were obtained from Yanbian University and acclimatized for 1 week before experimentation. Rats were fed a standard chow diet and kept under specific pathogen-free conditions at Yanbian University (Permit Number: SCXK [Ji] 2011-006). As previously described [13], isolated perfused beating left atria were prepared. For each atrium, transmural electrical field stimulation was applied with a luminal electrode at $1.5 \mathrm{~Hz}(0.3 \mathrm{~ms}, 30-40 \mathrm{~V})$, and the atrium was perfused with HEPES buffer using a peristaltic pump $(1.0 \mathrm{ml} / \mathrm{min})$ to enable atrial pacing for the measurement of pulse pressure changes. Oxygen was continuously supplied, and the temperature of the atrium was maintained at $36^{\circ} \mathrm{C}$. HEPES buffer contained (in $\mathrm{mM}) 118 \mathrm{NaCl}, 4.7 \mathrm{KCl}, 2.5 \mathrm{CaCl}_{2}, 1.2 \mathrm{MgCl}_{2}, 25 \mathrm{NaHCO}_{3}, 10.0$ glucose, and 10.0 HEPES (pH 7.4 with $\mathrm{NaOH}$ ), together with $0.1 \%$ bovine serum albumin.

\section{Acute hypoxic atrial model preparation}

To replace $\mathrm{O}_{2}$ with $\mathrm{N}_{2}$ to establish the acute hypoxic atria model, standard HEPES buffer was replaced with a $\mathrm{N}_{2}$-saturated HEPES buffer. Inhibitory atrial dynamics and significantly increased ANP secretion were the criteria used to indicate successful hypoxic atrial modeling.

\section{Determination of ANP and atrial pulse pressure}

As previously described [13], the levels of immunoreactive ANP in perfusates were measured with an Iodine $\left[{ }^{125} \mathrm{I}\right]$ Atrial Natriuretic Factor Radioimmunoassay Kit (North Institute of Biological Technology, Beijing, China). Intra-atrial pressure was recorded using a Physiograph (RM6240BD; Chengdu, China) via a pressure transducer (Statham P23Db; Oxnard, CA, USA).

\section{Experimental protocols}

All rats were randomly divided into: control, hypoxia only, BQ123/BQ788/BQ123 + BQ788 + hypoxia/ET-1, GLX351322 + hypoxia, ET-1 only, varespladib + hypoxia/ET-1, CAY10650 + hypoxia/ET-1, NAC + hypoxia, Src inhibitor $1+$ hypoxia, PD98059 + hypoxia, and LY294002 + hypoxia groups ( $\mathrm{n}=6$ per group).

Each atrium was perfused for 60 min to stabilize ANP secretion and atrial dynamics parameters. After two control cycles (12min experimental cycle), $\mathrm{O}_{2}$ was replaced with $\mathrm{N}_{2}$ and a hypoxic buffer was infused for four periods to observe changes in the atrial dynamics and ANP levels of the perfusates. Under a controlled temperature of $4^{\circ} \mathrm{C}$, perfusates were collected every $2 \mathrm{~min}$ to measure ANP levels. Immediately after perfusion, atrial tissue was frozen and stored at $-80^{\circ} \mathrm{C}$ for western blotting. Subsequently, another series of experiments were performed to investigate the mechanism of hypoxia-induced ANP secretion. After one control period, one treatment cycle was followed by four cycles of infusion of the treatment agent plus hypoxia. Treatment agents 
were as follows: BQ123 $(0.3 \mu \mathrm{M})$, BQ788 $(0.3 \mu \mathrm{M})$, ET-1 $(3.0 \mathrm{nM})$ GLX351322 $(35.0 \mu \mathrm{M})$, varespladib $(5.0 \mu \mathrm{M})$, CAY10650 (120.0 $\mathrm{nM})$, NAC (15.0 mM), Src inhibitor 1 (1.0 $\mu \mathrm{M})$, PD98059 (30.0 $\mu \mathrm{M})$, and LY294002 $(30.0 \mu \mathrm{M})$.

\section{Hydrogen peroxide assay}

The appropriate colorimetric assay kit (E-BC-K102-M; Elabscience, Wuhan, China) was used to measure the $\mathrm{H}_{2} \mathrm{O}_{2}$ in rat atrial tissue.

\section{Western blot analysis}

The expression of target proteins in left atrial tissues was determined by western blotting. Proteins were homogenized in radioimmunoprecipitation assay lysis buffer (Solarbio Institute of Biotechnology, Shanghai, China). Protein concentrations were determined using an Enhanced Bicinchoninic Acid Protein Assay Kit (Beyotime Institute of Biotechnology, Shanghai, China). Solubilized proteins were denatured in Lane Maker Loading Buffer (CWBio, Beijing, China), separated by sodium dodecyl sulfatepolyacrylamide gel electrophoresis on $8 \%$ gels, and transferred to polyvinylidene difluoride filter membranes (Beyotime Institute of Biotechnology). Membranes were blocked with 5\% nonfat dry milk in phosphate-buffered saline at room temperature. After $2 \mathrm{~h}$, membranes were incubated with primary antibodies at $4^{\circ} \mathrm{C}$ overnight. The following day, membranes were washed and incubated with a secondary antibody (AP132P; Nachuan Biotech, Changchun, China) for $2 \mathrm{~h}$ at room temperature. Bands were visualized with the ECL Plus Western Blotting Detection System (ECL Western Blot Kit, RB-SRCHLGT; Ray Biotech, Atlanta, GA, USA). The densitometry of western blots was quantified using ImageJ software (USA National Institute of Health, Bethesda, MA, USA).

\section{Statistical analysis}

Prism software (Version 3.0; GraphPad Software, San Diego, CA, USA) was used for statistical analyses. Student's t-test was performed to statistically compare groups, and one-way ANOVA followed by Dunnett's multiple comparison test was performed for multiple group analysis. Statistical significance was defined as $\mathrm{p}<0.05$. All data are presented as the mean \pm standard error of the mean (SEM).

\section{RESULTS}

\section{Effect of hypoxia on atrial ANP secretion and dynamics}

To verify the successful establishment of the atrial hypoxic model, ANP secretion and dynamics were observed using isolated perfused beating rat atria. The results showed that hypoxia potently stimulated ANP secretion ( $p<0.05$ vs. control; Fig. 1A) and concomitantly inhibited atrial dynamics ( $\mathrm{p}<0.05$ vs. control; Fig. 1B). The data demonstrated that the establishment of the acute hypoxic model of beating rat atria was successful.

\section{Effect of hypoxia on NOX2 and NOX4 expression and its role in ANP secretion}

To investigate the effect of hypoxia on NOX2 and NOX4 expression and its role in ANP secretion, a series of experiments were performed using isolated perfused beating rat atria. The results showed that hypoxia significantly upregulated the expression of NOX4 but not NOX2 ( $p<0.05$ vs. control; Fig. 2A and $\mathrm{B}$ ), which was completely blocked by the ETRA and ETRB antagonists $\mathrm{BQ} 123$ and $\mathrm{BQ788}$, respectively $(\mathrm{p}<0.05$ vs. hypoxia; Fig. 2B). In addition, hypoxia-induced ANP secretion was sub-
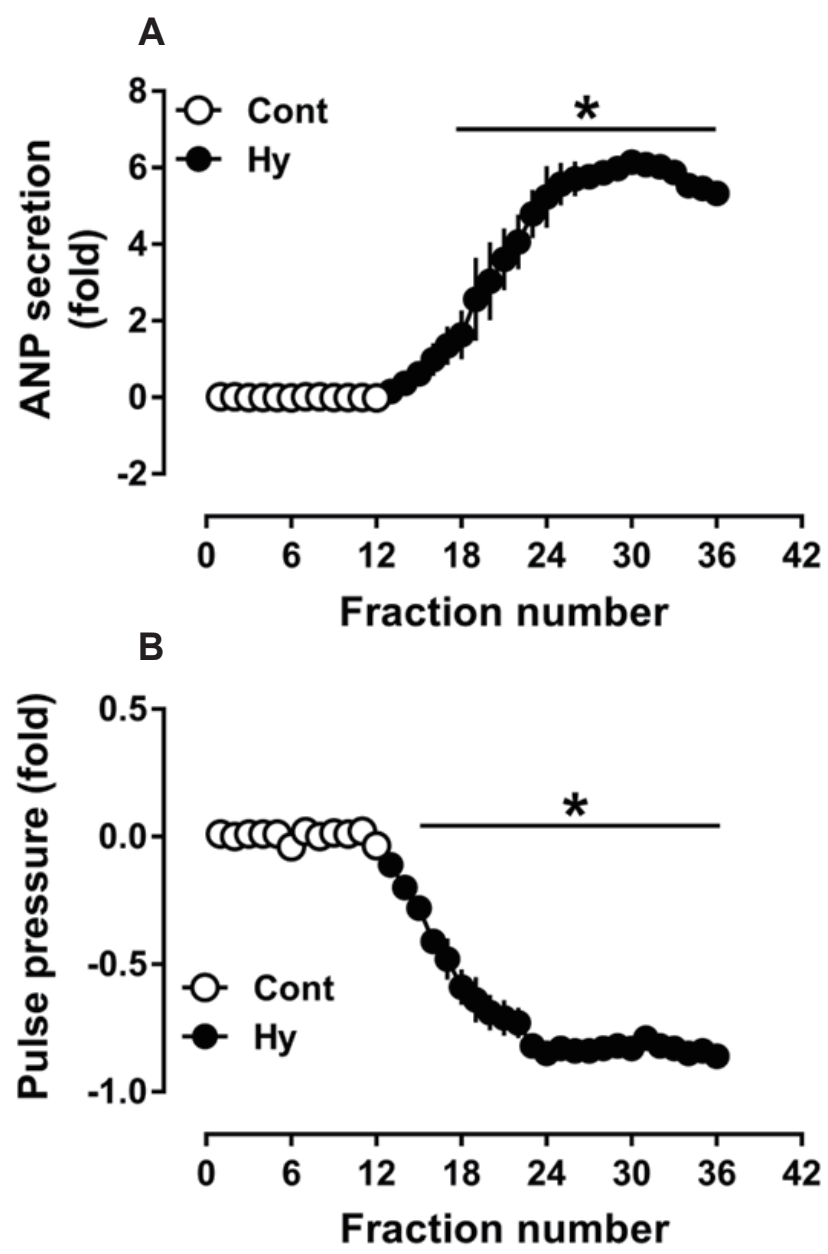

Fig. 1. Effect of hypoxia on ANP secretion (A) and dynamics (B) in isolated beating rat atria. Data were expressed as mean \pm standard error of the mean $(n=6)$. Cont, control; Hy, hypoxia. ${ }^{*} p<0.05$ vs. control. 

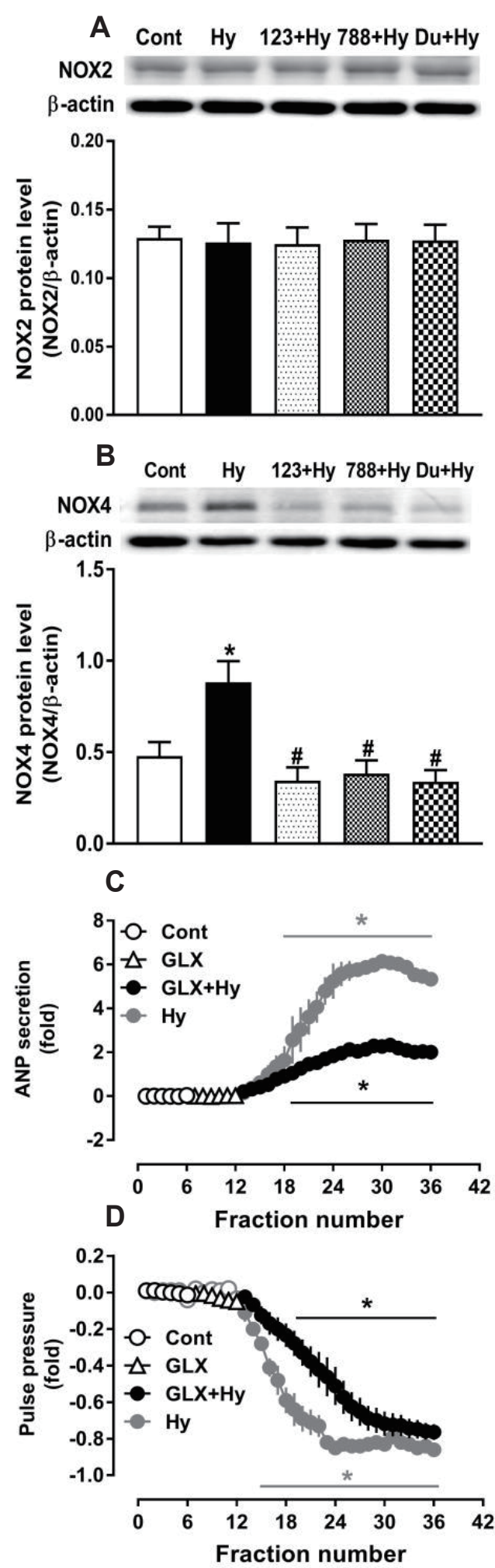

Fig. 2. Effect of hypoxia on NOX2 and NOX4 expression and its role in the regulation of ANP secretion in isolated beating rat atria. Data were expressed as the mean \pm standard error of the mean ( $A$ and $B, n=$ 5; $C$ and $D, n=6$ ). Cont, control; Hy, hypoxia; $123, B Q 123$, an antagonist of ETRA; 788, BQ788, an antagonist of ETRB; GLX, 351322, an antagonist of NOX4. ${ }^{*} p<0.05$ vs. control; ${ }^{\#} p<0.05$ vs. hypoxia. stantially attenuated by the NOX4 antagonist GLX351322 (p < 0.05 vs. control and hypoxia, respectively; Fig. 2C). The hypoxiainduced atrial dynamics were shifted to a rightward slightly by GLX351322, but it failed to significantly modulate the inhibitory effect of hypoxia on atrial dynamics ( $p<0.05$ vs. control; Fig. 2D). These results indicate that NOX4 regulated by endogenous ET-1 is involved in the regulation of ANP secretion in beating rat atria under hypoxic conditions.

\section{Effects of ET-1 and PLA2 on NOX4 expression under normoxic conditions}

To explore the mechanism of ET-1-induced NOX4 expression, exogenous ET-1 and antagonists of ETRs and PLA2 were used in the following experiments under normoxic conditions. The data showed that exogenous ET-1 evidently upregulated the expression of NOX4 ( $<0.05$ vs. control; Fig. 3A, B, and D), which was completely abolished by BQ123, BQ788, and the sPLA2 antagonist varespladib ( $<<0.05$ vs. ET-1; Fig. $3 \mathrm{~A}$ and B). Furthermore, varespladib significantly inhibited ET-1-induced cPLA2 expression ( $\mathrm{p}$ $<0.05$ vs. control and ET-1 respectively; Fig. 3C), and the cPLA2 antagonist CAT10650 evidently blocked the ET-1-induced expression of NOX4 ( $p<0.05$ vs. ET-1; Fig. 3D). These data demonstrate that ET-1 upregulated NOX4 expression through the activation of cPLA2 via SPLA2.

\section{Effect of hypoxia on $\mathrm{H}_{2} \mathrm{O}_{2}$ production and its role in ANP secretion}

To determine the effect of hypoxia on $\mathrm{H}_{2} \mathrm{O}_{2}$ production and its role in hypoxia-induced ANP secretion, another series of experiments were performed with antagonists of NOX4 and ROS. As shown in Fig. 4, hypoxia significantly increased the production of $\mathrm{H}_{2} \mathrm{O}_{2}$ ( $\mathrm{p}<0.05$ vs. control; Fig. 4A), and GLX351322 almost completely inhibited the hypoxia-increased production of $\mathrm{H}_{2} \mathrm{O}_{2}$ (p $<0.05$ vs. hypoxia; Fig. 4A). Moreover, the ROS antagonist NAC remarkably inhibited the hypoxia-induced ANP secretion ( $\mathrm{p}<$ 0.05 vs. control, $\mathrm{p}<0.05$ vs. hypoxia; Fig. 4B). These data suggest that $\mathrm{H}_{2} \mathrm{O}_{2}$ produced by NOX4 is associated with hypoxia-induced ANP secretion in beating rat atria.

\section{Effect of NOX4 on Src expression and its role in hypoxia-induced ANP secretion}

To clarify the downstream mechanism of NOX4 in hypoxiainduced ANP secretion, the effect of NOX4 on Src expression and its role in the regulation of ANP secretion were investigated. The results showed that hypoxia markedly increased Src expression ( $\mathrm{p}<0.05$ vs. control; Fig. $5 \mathrm{~A}-\mathrm{C}$ ), which was blocked not only by BQ123 and BQ788 ( $<0.05$ vs. hypoxia; Fig. $5 \mathrm{~A}$ ) but also GLX351322 and NAC ( $<<0.05$ vs. hypoxia; Fig. 5B and C). Additionally, exogenous ET-1 significantly increased the expression of 


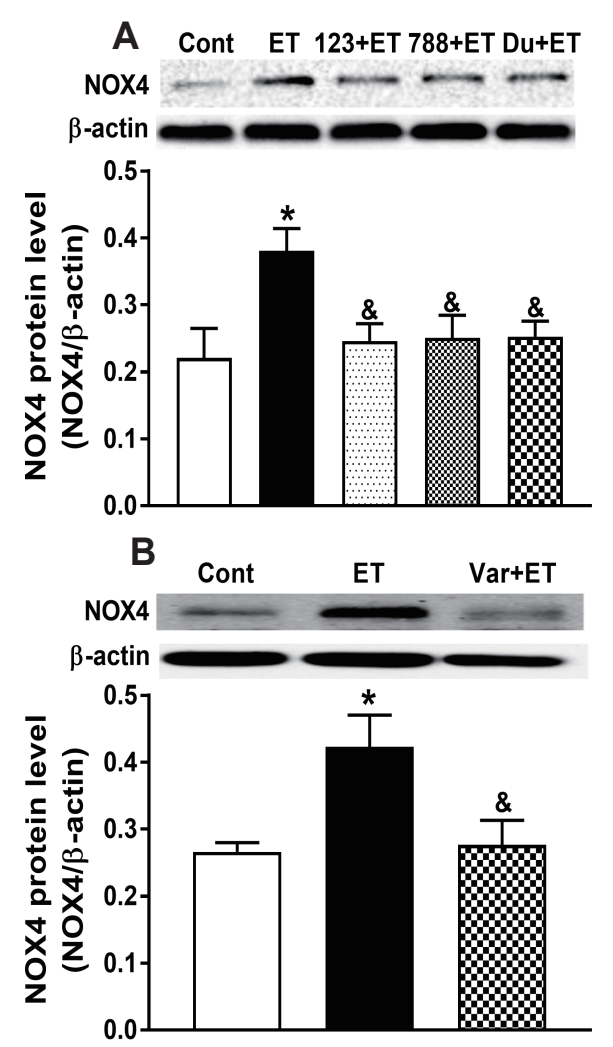

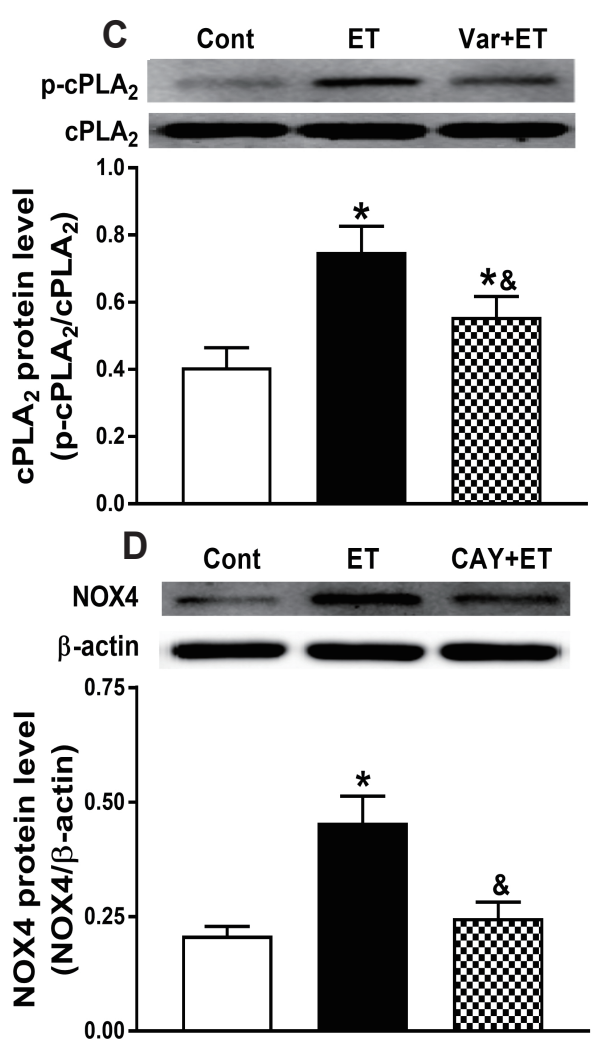

Fig. 3. Effects of ETR and PLA2 antagonists on NOX4 (A, B \& D) and pCPLA2 (C) expression in beating rat atria under normoxic conditions. Data were expressed as the mean \pm standard error of the mean $(n=5)$. Cont, control; ET, ET-1; Var, varespladib, an antagonist of sPLA2; CAY, CAY10650, an antagonist of $c$ PLA2. ${ }^{*} p<0.05$ vs. control; ${ }^{\&} p<0.05$ vs. ET-1.
Src, and this effect was inhibited by NAC ( $\mathrm{p}<0.05 \mathrm{vs}$. control and ET-1, respectively; Fig. 5D). These results reveal that ET-1/NOX4/ ROS signaling was involved in the regulation of Src expression.

\section{Effect of Src on ERK1/2 and Akt expression and its role in regulating GATA4 levels}

To elucidate the mechanism by which Src regulates hypoxiainduced ANP secretion, the effect of Src on ERK1/2 and Akt expression and its role in the regulation of GATA4 levels were evaluated. The results indicated that hypoxia significantly upregulated the expression of ERK1/2 and Akt ( $p<0.05$ vs. control; Fig. 6A and B, respectively), but these effects were blocked by Src inhibitor 1 ( $\mathrm{p}<0.05$ vs. hypoxia; Fig. 6A and B, respectively). Hypoxia also markedly increased the levels of GATA4 ( $p<0.05$ vs. control; Fig. 6C), which was substantially inhibited by ERK1/2 and Akt antagonists (PD98059 and LY294002, p < 0.05 vs. hypoxia, respectively; Fig. 6C). Furthermore, Src inhibitor 1 significantly attenuated hypoxia-induced ANP secretion ( $\mathrm{p}<0.05$ vs. control, $\mathrm{p}<0.05$ vs. hypoxia, respectively; Fig. 6D). These results demonstrate that Src upregulated the expression of GATA4 through the activation of ERK1/2 and Akt signaling to regulate hypoxiainduced ANP secretion in beating rat hypoxic atria.

\section{DISCUSSION}

The present study shows that atrial NOX4 expression was upregulated by ET-1 under both hypoxic and normoxic conditions, leading to the activation of Src. Activated Src subsequently increased GATA4 expression via ERK1/2 and Akt signaling to regulate hypoxia-induced ANP secretion in isolated perfused beating rat atria.

NOX4, a main source of ROS production, is expressed in the heart and is overexpressed in response to hypoxia, myocardial ischemia, and pressure overload; however, its role in cardiomyocytes remains unclear $[14,15]$. NOX activity is stimulated by various GPCR agonists, including angiotensin II, ET-1, thrombin, ATP, and thromboxane [12]. In this study, hypoxia evidently upregulated the expression of NOX4, and this effect was completely abolished by the ET-1 receptor antagonists BQ123 and BQ788. In addition, exogenous ET-1 significantly upregulated the expression of NOX4 and concomitantly activated cPLA2; however, ET-1-augmented NOX4 expression was inhibited by BQ123 and BQ788 in normoxic beating rat atria. The sPLA2 antagonist varespladib also blocked the effect of ET-1-induced NOX4 expression through the inhibition of ET-1-activated CPLA2, and the CPLA2 antagonist CAY10650 mimicked the role of varespladib in regulating the expression of NOX4 induced by ET-1. ET-1 promotes the activation of tumor necrosis factor alpha [16], leading to enhanced sPLA2 transcription levels in isolated neonatal rat cardiomyocytes [17]. Therefore, our results indicate that ET-1 upregulates 


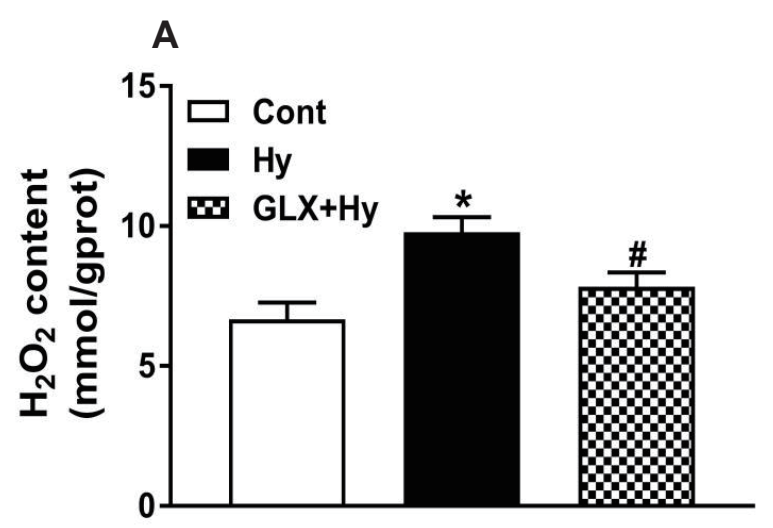

B

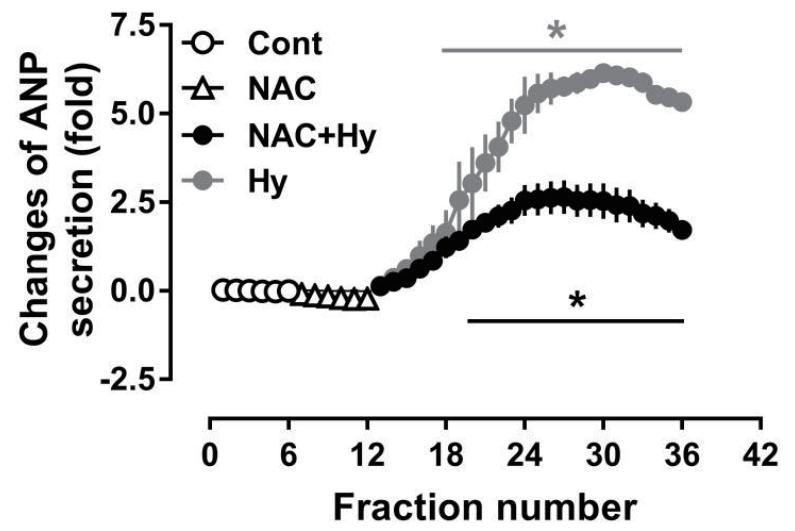

Fig. 4. Effect of hypoxia on $\mathrm{H}_{2} \mathrm{O}_{2}$ production and its role in ANP secretion in beating rat atria. Data were expressed as the mean \pm standard error of the mean ( $A, n=5 ; B, n=6)$. Cont, control; Hy, hypoxia; GLX, GLX351322, an antagonist of NOX4; NAC, N-Acetyl-D-cysteine, an antagonist of ROS. ${ }^{*} \mathrm{p}<0.05$ vs. control; ${ }^{*} \mathrm{p}<0.05$ vs. hypoxia.

NOX4 expression through the activation of cPLA2 via sPLA2. These results are consistent with the previous studies mentioned above and support previous reports that ET-1 is regulated by NOX4 activity via ETA in endothelial cells [18] and ETB, leading to neurodegeneration via NOX-driven superoxide formation [19]. Furthermore, our results showed that hypoxia potently promoted ANP secretion, increased ROS production, and inhibited atrial dynamics. Hypoxia-induced ANP secretion was markedly attenuated by NOX4 and ROS antagonists, and hypoxia-induced atrial dynamics were slightly shifted rightward by treatment with a ROS antagonist without significant modulation of the inhibitory effect of hypoxia on atrial dynamics. These results indicate that hypoxia-induced NOX4 is modulated by endogenous ET-1 via its receptors to regulate hypoxia-induced ANP secretion. Our findings support a previous report that demonstrated an association of increased NOX4 activity in response to a high pacing frequency with increased ANP secretion in rat atria [20].

Src is a non-receptor protein-tyrosine kinase that plays important roles in cell growth, division, migration, and survival signaling pathways [21]. GPCRs or hypoxia can stimulate Src and further activate downstream signaling, such as ERK1/2 and Akt

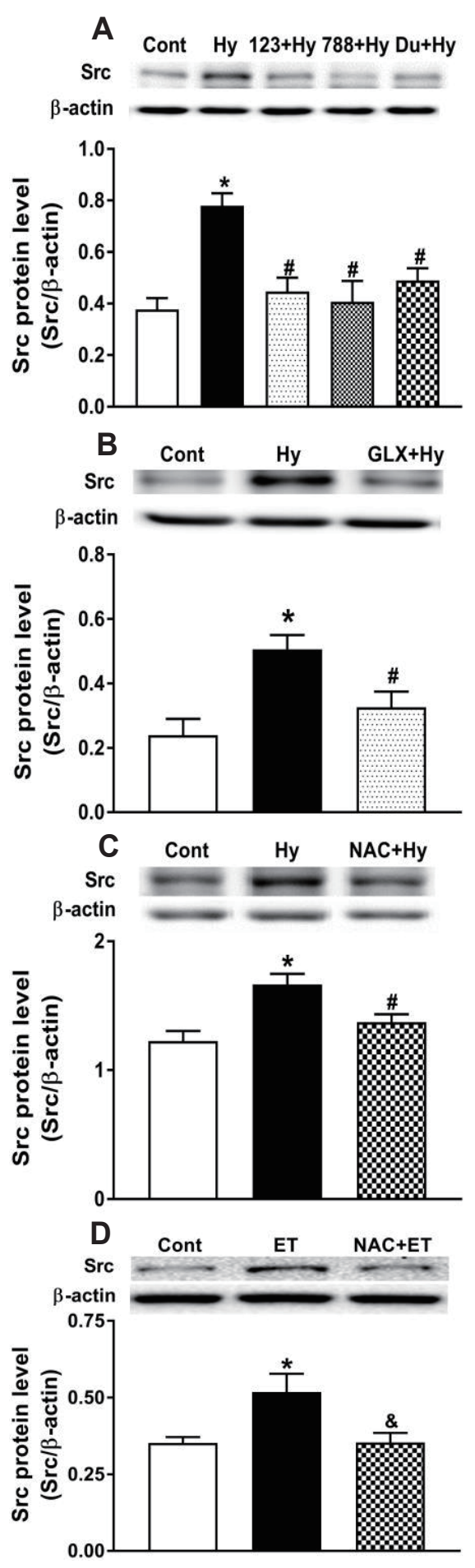

Fig. 5. Effects of ET-1, NOX4 and ROS on Src expression in beating rat atria under hypoxic (A-C) and normoxic (D) conditions. Data were expressed as the mean \pm standard error of the mean $(n=5)$. Cont, control; Hy, hypoxia; GLX, GLX351322; NAC, N-Acetyl-D-cysteine; ET, ET1. ${ }^{*} p<0.05$ vs. control; ${ }^{*} p<0.05$ vs. hypoxia; ${ }^{\&} p<0.05$ vs. ET-1.

pathways [12,22]. The results of this study also show that hypoxia notably upregulated the expression of Src, which was completely blocked by antagonists of ETRs, NOX4, and ROS. In addition, exogenous ET-1 upregulated Src expression, and this was inhib- 


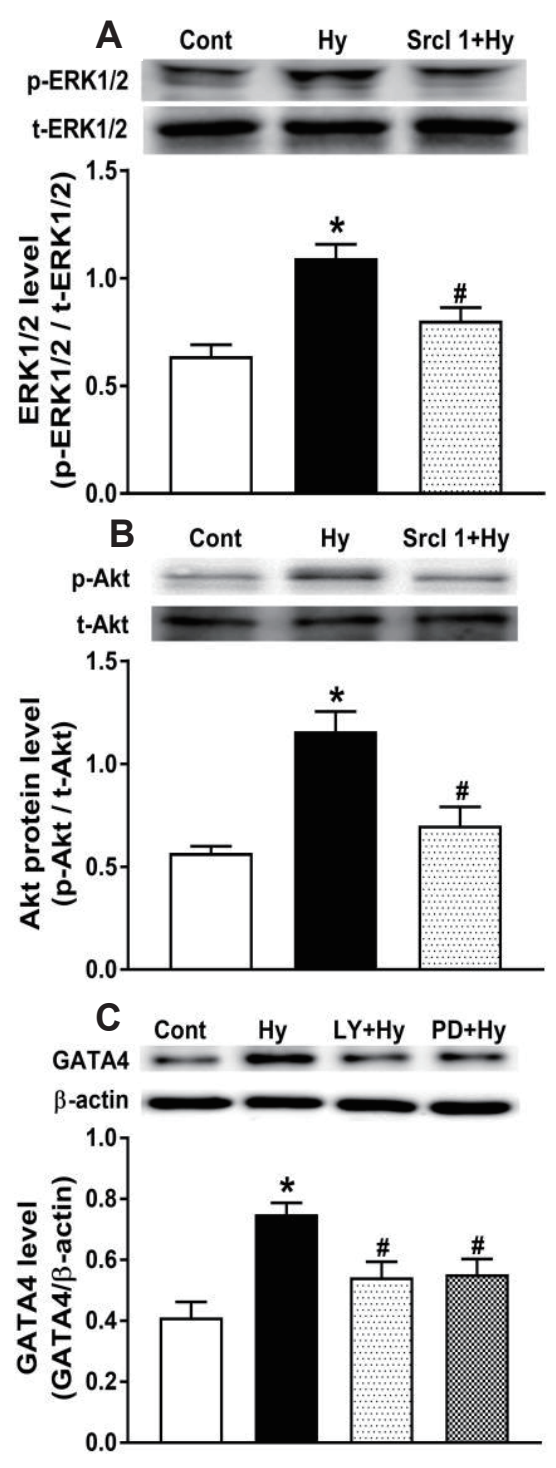

D

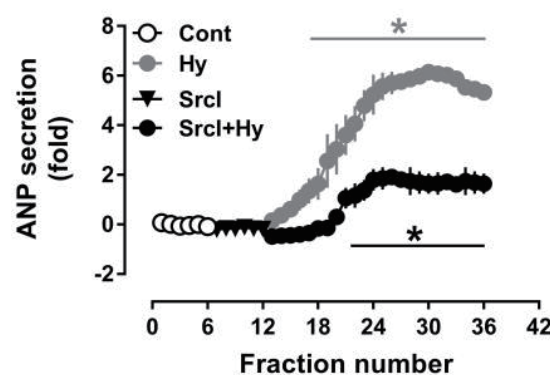

Fig. 6. Effect of Src on ERK1/2 and Akt expression (A, B) and its role in the regulation of GATA4 (C) and ANP secretion (D) in hypoxic beating rat atria. Data were expressed as the mean \pm standard error of the mean ( $A-C, n=5 ; D, n=6$ ). Cont, control; Hy, hypoxia; Srcl, Src inhibitor 1; PD, PD98059, an antagonist of ERK1/2; LY, LY294002, an antagonist of Akt. ${ }^{*} p<0.05$ vs. control; ${ }^{*} p<0.05$ vs. hypoxia.

ited by the ROS antagonist NAC in normoxic beating rat atria. These results indicate that NOX4 is regulated by ET-1 to activate Src by increasing the production of ROS. Moreover, a Src inhibi- tor significantly attenuated hypoxia-induced ANP secretion, thus mimicking the inhibitory effect of NOX4 antagonism on hypoxia-induced ANP secretion. These results demonstrate an association between the regulation of Src by ET-1/NOX4 signaling and increased secretion of ANP under hypoxic conditions. Furthermore, our results showed that the hypoxia-induced upregulation of ERK1/2 and Akt expression was blocked by a Src antagonist and that hypoxia-induced GATA4 expression was substantially inhibited by ERK1/2 and Akt antagonists. These results indicate that the Src-mediated regulation of ERK1/2 and Akt signaling modulates hypoxia-induced ANP secretion through the activation of GATA4 in beating rat atria. Previous studies confirmed the involvement of ERK and Akt signaling pathways in the regulation of hypoxia-induced ANP secretion [23], and GATA4 is an important transcription factor for ANP gene transcription [24,25]. Our current findings support these results.

In summary, hypoxia-induced NOX4/Src signaling controlled by endogenous ET-1 via ETRs increased GATA4 expression through the activation of ERK1/2 and Akt pathways, ultimately mediating the regulation of ANP secretion by hypoxia in isolated beating rat atria.

\section{ACKNOWLEDGEMENTS}

This work was supported by the National Natural Science Foundation of China (No. 81660089; 81960099). We thank Melissa Crawford, PhD, Liwen Bianji, Edanz Group China (www. liwenbianji.cn/ac), for editing the English text of a draft of this manuscript.

\section{CONFLICTS OF INTEREST}

The authors declare no conflicts of interest.

\section{REFERENCES}

1. Zhang Y, Murugesan P, Huang K, Cai H. NADPH oxidases and oxidase crosstalk in cardiovascular diseases: novel therapeutic targets. Nat Rev Cardiol. 2020;17:170-194.

2. Zhang M, Perino A, Ghigo A, Hirsch E, Shah AM. NADPH oxidases in heart failure: poachers or gamekeepers? Antioxid Redox Signal. 2013;18:1024-1041.

3. Bedard K, Krause KH. The NOX family of ROS-generating NADPH oxidases: physiology and pathophysiology. Physiol Rev. 2007;87:245313.

4. Bendall JK, Cave AC, Heymes C, Gall N, Shah AM. Pivotal role of a gp91(phox)-containing NADPH oxidase in angiotensin II-induced cardiac hypertrophy in mice. Circulation. 2002;105:293-296.

5. Rodiño-Janeiro BK, Paradela-Dobarro B, Castiñeiras-Landeira MI, Raposeiras-Roubín S, González-Juanatey JR, Alvarez E. Current status of NADPH oxidase research in cardiovascular pharmacology. 
Vasc Health Risk Manag. 2013;9:401-428.

6. Cho KW, Seul KH, Ryu H, Kim SH, Koh GY. Characteristics of distension-induced release of immunoreactive atrial natriuretic peptide in isolated perfused rabbit atria. Regul Pept. 1988;22:333-345.

7. McGrath MF, de Bold ML, de Bold AJ. The endocrine function of the heart. Trends Endocrinol Metab. 2005;16:469-477.

8. De Vito P, Incerpi S, Pedersen JZ, Luly P. Atrial natriuretic peptide and oxidative stress. Peptides. 2010;31:1412-1419.

9. Arjamaa O, Nikinmaa M. Hypoxia regulates the natriuretic peptide system. Int J Physiol Pathophysiol Pharmacol. 2011;3:191-201.

10. Wang D, Gladysheva IP, Fan TH, Sullivan R, Houng AK, Reed GL. Atrial natriuretic peptide affects cardiac remodeling, function, heart failure, and survival in a mouse model of dilated cardiomyopathy. Hypertension. 2014;63:514-519.

11. Hong L, Xi J, Zhang Y, Tian W, Xu J, Cui X, Xu Z. Atrial natriuretic peptide prevents the mitochondrial permeability transition pore opening by inactivating glycogen synthase kinase $3 \beta$ via PKG and PI3K in cardiac H9c2 cells. Eur J Pharmacol. 2012;695:13-19.

12. MacKay CE, Knock GA. Control of vascular smooth muscle function by Src-family kinases and reactive oxygen species in health and disease. J Physiol. 2015;593:3815-3828.

13. Li X, Han ZN, Liu Y, Hong L, Cui BR, Cui X. Endogenous ET-1 promotes ANP secretion through activation of COX2-L-PGDS-PPAR $\gamma$ signaling in hypoxic beating rat atria. Peptides. 2019;122:170150.

14. Kuroda J, Ago T, Matsushima S, Zhai P, Schneider MD, Sadoshima J. NADPH oxidase 4 (Nox4) is a major source of oxidative stress in the failing heart. Proc Natl Acad Sci U S A. 2010;107:15565-15570.

15. Zhang M, Brewer AC, Schröder K, Santos CX, Grieve DJ, Wang M, Anilkumar N, Yu B, Dong X, Walker SJ, Brandes RP, Shah AM. NADPH oxidase- 4 mediates protection against chronic load-induced stress in mouse hearts by enhancing angiogenesis. Proc Natl Acad Sci U S A. 2010;107:18121-18126.

16. Ruetten H, Thiemermann C. Endothelin-1 stimulates the biosynthesis of tumour necrosis factor in macrophages: ET-receptors, signal transduction and inhibition by dexamethasone. J Physiol Pharmacol. 1997;48:675-688.

17. De Windt LJ, Willemsen PH, Pöpping S, Van der Vusse GJ, Reneman RS, Van Bilsen M. Cloning and cellular distribution of a group II phospholipase A2 expressed in the heart. J Mol Cell Cardiol. 1997;29:2095-2106.

18. Su J, An XR, Li Q, Li XX, Cong XD, Xu M. Improvement of vascular dysfunction by argirein through inhibiting endothelial cell apoptosis associated with ET-1/Nox4 signal pathway in diabetic rats. Sci Rep. 2018;8:12620.

19. Tam SW, Feng R, Lau WK, Law AC, Yeung PK, Chung SK. Endothelin type $\mathrm{B}$ receptor promotes cofilin rod formation and dendritic loss in neurons by inducing oxidative stress and cofilin activation. $J$ Biol Chem. 2019;294:12495-12506.

20. Gao S, Yuan K, Shah A, Kim JS, Park WH, Kim SH. Suppression of high pacing-induced ANP secretion by antioxidants in isolated rat atria. Peptides. 2011;32:2467-2473.

21. Roskoski R Jr. Src protein-tyrosine kinase structure, mechanism, and small molecule inhibitors. Pharmacol Res. 2015;94:9-25.

22. Yuan Z, McCauley R, Chen-Scarabelli C, Abounit K, Stephanou A, Barry SP, Knight R, Saravolatz SF, Saravolatz LD, Ulgen BO, Scarabelli GM, Faggian G, Mazzucco A, Saravolatz L, Scarabelli TM. Activation of Src protein tyrosine kinase plays an essential role in urocortin-mediated cardioprotection. Mol Cell Endocrinol. 2010;325:1-7.

23. Zhang QL, Cui BR, Li HY, Li P, Hong L, Liu LP, Ding DZ, Cui X. MAPK and PI3K pathways regulate hypoxia-induced atrial natriuretic peptide secretion by controlling HIF-1 alpha expression in beating rabbit atria. Biochem Biophys Res Commun. 2013;438:507512.

24. Hayek S, Nemer M. Cardiac natriuretic peptides: from basic discovery to clinical practice. Cardiovasc Ther. 2011;29:362-376.

25. Temsah R, Nemer M. GATA factors and transcriptional regulation of cardiac natriuretic peptide genes. Regul Pept. 2005;128:177-185. 